\section{Spontaneous Cutaneous Endometriosis of the Umbilicus: A Case Report}

\section{Abstract}

Cutaneous endometriosis represents an unusual ectopic growth of endometrial tissue outside the uterine cavity. We present a case of spontaneous (primary) cutaneous endometriosis of the umbilicus with review of the appropriate literature. Our patient is a 30 -year-old African American female, with no previous surgical history, who presented to our dermatology clinic with concern regarding nodules in her umbilicus. According to the patient, the nodules were associated with cyclical pain, episodic bleeding and fluctuation in size. Dermatological examination revealed multiple 5-6 $\mathrm{mm}$ reddish-blue, dome shaped papules within the umbilicus. Following shave biopsy, histological examination revealed endometrial glands surrounded by fibrotic, mucinous stroma and erythrocyte extravasation with hemosiderin deposition. Also identified, were ductal structures with cilia on the internal lining. The lesions were diagnosed as cutaneous endometriosis with cilia. The patient was referred to plastic surgery for local excision and gynecology for assessment of endometriosis in the pelvic cavity.

Keywords: Spontaneous cutaneous endometriosis; Umbilicus; Endometrial glands; Cilia

Received: April 16, 2017; Accepted: May 08, 2017; Published: May 16, 2017

\section{Katherine Braunlich', Gregory Delost ${ }^{2}$, Kord Honda ${ }^{2}$ and Jenifer Lloyd ${ }^{2}$}

\author{
1 Largo Medical Center, Largo, Florida \\ 2 Department of Dermatology, University \\ Hospitals Cleveland, Ohio
}

\section{Corresponding author:}

Katherine Braunlich

$\equiv$ kbraunlich2@gmail.com

DO, Largo Medical Center, Largo, Florida-33778, USA.

Tel: (219) 508-5671

Citation: Braunlich K, Delost G, Honda K, et al. Spontaneous Cutaneous Endometriosis of the Umbilicus: A Case Report. J Rare Disord Diagn Ther. 2017, 3:3.

\section{Introduction}

Cutaneous endometriosis represents a non-neoplastic, ectopic growth of endometrial glands and stroma outside the uterine cavity [1-4]. Daneil Schroen, a German physician, first described endometriosis in 1690 [5]. Years later, in 1885, the first case of cutaneous endometriosis was identified and reported [3]. Cutaneous endometriosis is typically divided into two categories, scar endometriosis and spontaneous (primary) cutaneous endometriosis.

Traditionally, cutaneous endometriosis is an entity known to arise within surgical scars following abdominal or gynecologic procedures $[3,6]$. This form of cutaneous endometriosis is often referred to as scar endometriosis. Cesarean section is the most common preceding surgical procedure $[3,7]$. Endometriosis arising in a surgical scar is proposed to be the result of iatrogenic transplantation with microscopic seeding $[2,5,8,9]$.

Spontaneous cutaneous endometriosis, also known as primary cutaneous endometriosis, is far less common and accounts for approximately (30\%) of all cases of cutaneous endometriosis [8]. Spontaneous cutaneous endometriosis of the umbilicus is known as a Villar nodule, first described by Dr. Villar in $1886[1,5,10]$.
Theories postulate that the umbilicus may act as a physiological scar, explaining the development of spontaneous disease in this location [6]. The precise pathogenesis of spontaneous cutaneous endometriosis however, remains an enigma. Overall, umbilical endometriosis is relatively uncommon and represents only $0.5-1 \%$ of all cases of ectopic endometrial tissue $[8,9,11]$. We present an unusual case of spontaneous (primary) cutaneous endometriosis with cilia in the umbilicus of a nulliparous woman with no history of surgical intervention.

\section{Case Report}

A 30-year-old Gravida 0 Para 0 African-American female presented to the dermatology clinic with the chief complaint of painful and pruritic umbilical lesions. She had visited numerous physicians with this complaint and was most recently diagnosed with contact dermatitis. After nickel avoidance and topical corticosteroids yielded no improvement the patient was referred to dermatology. According to the patient, the lesions appeared 9 months prior after discontinuing Depo-Provera in an attempt to become pregnant. The patient also endorsed episodic bleeding, fluctuation in lesion size and dyspareunia. She was able to correlate the timing of the umbilical lesion bleeding to menstruation. 
She denied any menstrual cycle irregularities, history of endometriosis, history of chronic disease, allergies, hospitalizations or past surgical history including abdominal and gynecological surgeries. Additionally, she denied a history of smoking, alcohol, or recreational drug use. She was not taking any prescription medications or over the counter supplements at the time of the visit. A comprehensive review of systems was negative. Her family history was noncontributory.

Physical exam revealed multiple 5-6 $\mathrm{mm}$ reddish-blue, dome shaped papules within the umbilicus (Figure 1). The remainder of her skin exam was clear. There was no hepatosplenomegaly or lymphadenopathy. Routine Complete Blood Count (CBC), Basic Metabolic Profile (BMP) and Urine Analysis (UA) were all within normal limits.

Following a shave biopsy, histological examination revealed evidence of endometrial glands surrounded by fibrotic and mucinous stroma, erythrocyte extravasation with hemosiderin deposition and ductal structures with cilia on the internal lining (Figures $\mathbf{2}$ and $\mathbf{3}$ ).

The patient was referred to plastic surgery for local excision of the lesions and gynecology for assessment of additional endometriosis in the pelvic cavity.

\section{Discussion}

Cutaneous endometriosis is most commonly diagnosed in women between the ages of 31 and 38 years [1,3,5,12-14]. The disease should be suspected in any female presenting with cyclical pain, pruritus or episodic bleeding originating from a previous surgical scar, the inguinal region or the umbilicus $[3,9]$. These symptoms are likely the result of extravasation of erythrocytes and inflammatory infiltrate into surrounding tissue [9]. While cutaneous endometriosis is known to have exceedingly variable clinical presentations, the current literature seems to agree on some commonly noted symptoms. These symptoms include cyclical pain, sporadic bleeding and a palpable mass $[1,3]$. The most common physical findings include pigmented (i.e., red, brown, blue, purple) or skin colored papules or multilobulated

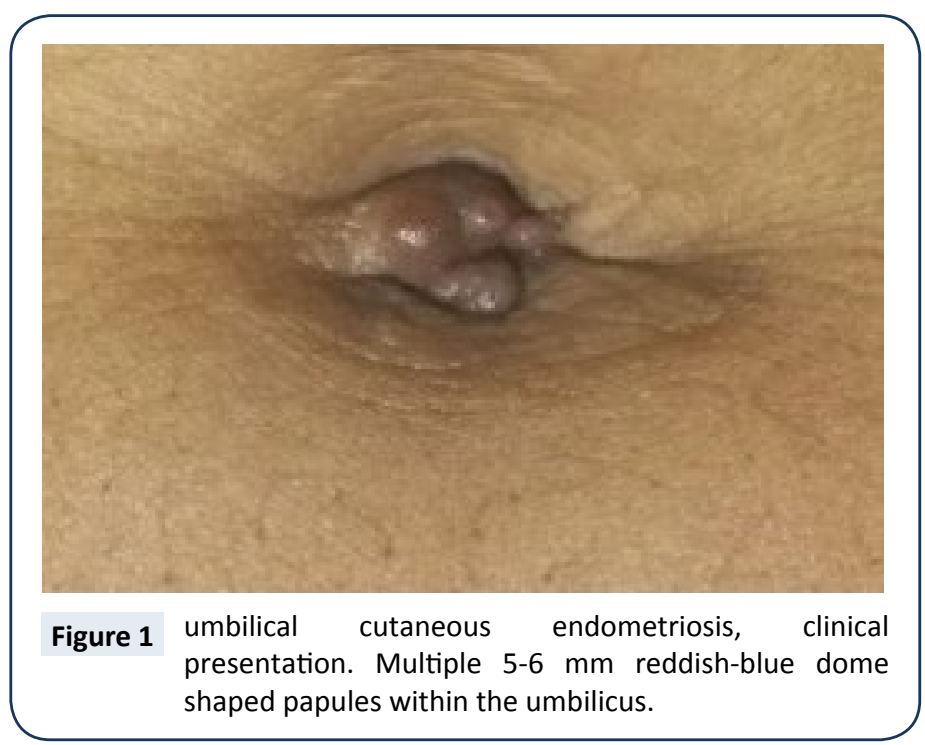

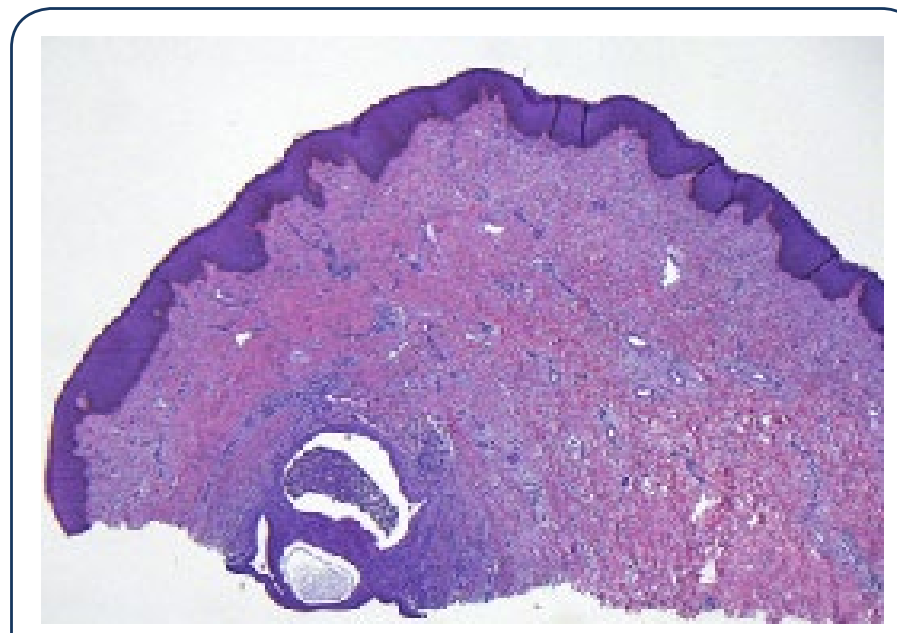

Figure 2 Hematoxylin and eosin statin of the resected tissue on low power. 4X magnification displaying, endometrial glands and fibrotic, mucinous stroma in the dermis.

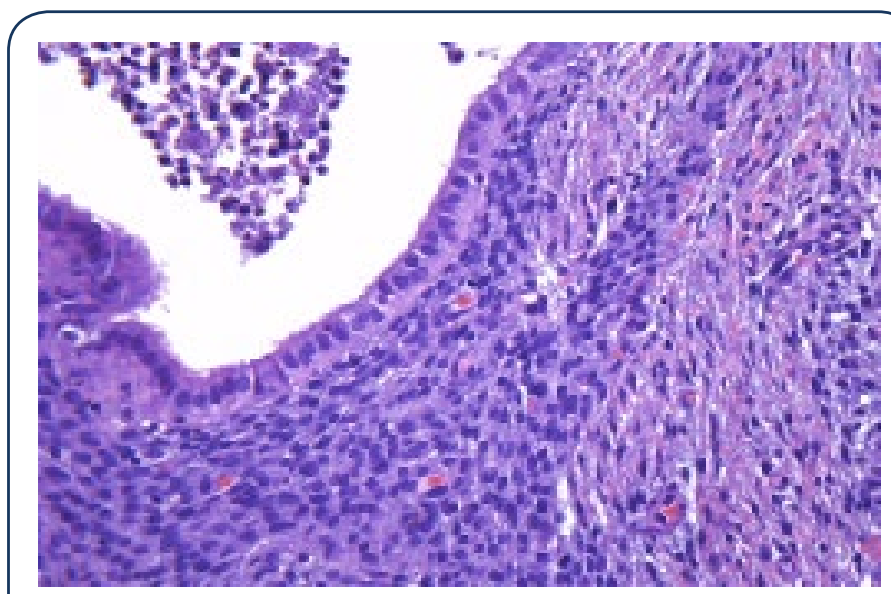

Figure $3 \mathrm{H} \& \mathrm{E}$ statin of the resected tissue on high power. 40X magnification, high power view, displaying cilia on the internal lining and erythrocyte extravasation with hemosiderin deposition.

nodules $[1,5]$. The majority of the cutaneous lesions range from 1.0 to $9.0 \mathrm{~cm}[1,5,13,15]$. Dermoscopy findings found to correlate with the disease include homogenous reddish pigmentation and small, deep hued, globular structures known as red atolls [5].

Individuals with spontaneous cutaneous endometriosis have a greater risk of coexisting endometriosis in the pelvic cavity [14]. As a result, patients may complain of associated dysmenorrhea or dyspareunia [15]. Those with spontaneous development of disease, like our patient, are more likely to have advanced pelvic disease in comparison to those with scar endometriosis $[3,14]$. When left untreated, a risk of malignant transformation exists. The most common form of malignant transformation, in cutaneous endometriosis, is clear-cell carcinoma [2,16]. For this reason, malignancy must be in the differential for any patient with changes in growth pattern or recurrent lesions.

Cutaneous endometriosis presents a diagnostic challenge due to its paucity and variable clinical presentation. Lack of awareness of this condition results in delayed and altogether missed diagnoses. 
The clinical differential diagnosis includes a keloid, hypertrophic scar, and metastatic carcinoma (Sister Mary Joseph's nodule). There are reports of cutaneous endometriosis presenting to general surgeons because of clinical manifestations resembling an incisional hernia, papilloma or other surgical ailment [17]. Prior to surgical removal, only (25\%) of cases receive a correct diagnosis of cutaneous endometriosis [2]. A retrospective study conducted by Douglas et al., found the average time to receive the correct diagnosis of cutaneous endometriosis was 24.54 months [14]. Agarwal et al., revealed the average time between onset of symptoms and surgical treatment is 31.7 months [3].

The characteristic histological feature is endometrial glands in the proliferative, secretory or decasualization phase within the dermis with erythrocyte extravasation or hemosiderin deposition $[2,5,9]$. Perls stain can be utilized to confirm the presence of hemosiderin but is not required to make the diagnosis [5]. Immunohistochemically, the cells stain with antibodies against CD10, cyclooxygenase-2 (COX-2), estrogen and progesterone receptor though these findings are not specific [4]. The differential diagnosis histologically includes endosalpingiosis. The latter typically has cilia and mucin containing cells, but lacks the hemosiderin and hemorrhage in the surrounding stroma. Our case is quite unusual in the finding of cilia within endometriosis.
The mainstay of treatment is wide local surgical excision $[3,5,8]$. The preferred timing for excision is at the end of the menstrual cycle when the glands are smallest, in an attempt to minimize the surgical defect $[2,5,9]$. Surgical excision is considered adequate therapy and patients should be reassured that recurrence and malignant transformation are quite rare. An additional component of treatment must include a referral to a gynecologist to evaluate for pelvic endometriosis.

\section{Conclusion}

Cutaneous endometriosis should be considered in the differential for all female patients who present with cyclical pain and an umbilical mass regardless of surgical history. We recommend biopsy prior to further treatment. Histologically, endometrial glands with a mucinous stroma, erythrocyte extravasation and hemosiderin deposition are seen and rarely cilia may be identified.

In summary, we herein present a case of spontaneous cutaneous endometriosis of the umbilicus. The clinical presentation was characteristic of cutaneous endometriosis but the histopathological examination was quite unusual with the presence of cilia. 


\section{References}

1 Stojanovic M, Brasanac D, Stojicic M (2013) Cutaneous inguinal scar endosalpingiosis and endometriosis. Am J Dermatopathol 35: 254-260.

2 Din A, Verjee L, Griffiths M (2013) Cutaneous endometriosis: A plastic surgery perspective. J Plast Reconstr Aesthet Surg 66: 129-130.

3 Agarwal A, Fong Y (2008) Cutaneous endometriosis. Singapore Med J 49: 704-709.

4 Terada S, Miyata Y, Nakazawa H (2006) Immunohistochemical analysis of an ectopic endometriosis in the uterine round ligament. Diagn Pathol 1: 27.

5 Kyamidis K, Lora V, KanitakisJ(2011) Spontaneous cutaneous umbilical endometriosis: Report of a new case with immunohistochemical study and literature review. Dermatol Online J 17: 5.

6 Steck D, Helwig E (1965) Cutaneous endometriosis. JAMA 191: 167-170.

7 Leite DE, Carvalho L, Korkes H (2009) Scar endometrioma following obstetric surgical inscicions: Retrospective study on 33 cases and review of the literature. Sao Paulo Med J 127: 270-277.

8 Rossari S, Grazzini M, Savarese I (2013) A particularly bothersome umbilical nodule. Indian J Dermatol Venereol Leprol 58: 330.

9 Fernandez V, Armario H, Cuevas S (2010) Cutaneous endometriosis. Int J Dermatol 49: 12.
10 Chatzikokkinou P, Thorfin J, Angelidis IK (2009) Spontaneous endometriosis in an umbilical skin lesion. Acta Dermatovenerologica Alpina 18: 126-130.

11 Fernandes H, Pailoor K, Marla N (2011) Primary umbilical endometriosis-Diagnosis by fine needle aspiration. J Ctyol 28: 214

12 Singh K, Lessells A, Adam DJ (1995) Presentation of endometriosis to general surgeons: a 10-year experience. Br J Surg 82: 1349-1351.

13 Victory R, Diamond M, Johns D (2007) Villar's nodule: A case report and systematic literature review of endometriosis externa of the umbilicus. J Minim Invasive Gynecol 14: 23-32.

14 Douglas C, Rotimi O (2004) Extragenital endometriosis a clinicopathological review of a Glasgow hospital experience with case illustrations. J Obstet Gynaecol 24: 804-808.

15 Costa I, Gomes C, Morais O (2013) Cutaneous endometriosis: Dermoscopic findings related to phases of the female hormonal cycle. Int J Dermatol 53: 2.

16 Ahn G, Scully R (1991) Clear cell carcinoma of the inguinal region arising from endometriosis. CA J 67:116-20.

17 Singh A (2012) Umbilical endometriosis mimicking as papilloma to general surgeons: A case report. Australas Med J 5: 272-274. 